\title{
Discrete Hamilton-Jacobi Theory and Discrete Optimal Control
}

\author{
Tomoki Ohsawa, Anthony M. Bloch, and Melvin Leok
}

\begin{abstract}
We develop a discrete analogue of HamiltonJacobi theory in the framework of discrete Hamiltonian mechanics. The resulting discrete Hamilton-Jacobi equation is discrete only in time. The correspondence between discrete and continuous Hamiltonian mechanics naturally gives rise to a discrete analogue of Jacobi's solution to the Hamilton-Jacobi equation. We prove discrete analogues of Jacobi's solution to the Hamilton-Jacobi equation and of the geometric HamiltonJacobi theorem. These results are readily applied to the discrete optimal control setting, and some well-known results in discrete optimal control theory, such as the Bellman equation, follow immediately. We also apply the theory to discrete linear Hamiltonian systems, and show that the discrete Riccati equation follows as a special case.
\end{abstract}

\section{INTRODUCTION}

\section{A. Discrete Mechanics}

Discrete mechanics is a reformulation of Lagrangian and Hamiltonian mechanics with discrete time, as opposed to a discretization of the equations in the continuous-time theory. It not only provides a systematic view of structurepreserving integrators, but also has interesting theoretical aspects analogous to continuous-time Lagrangian and Hamiltonian mechanics [see, e.g., 15; 17; 18]. The main feature of discrete mechanics is its use of discrete versions of variational principles. Namely, discrete mechanics assumes that the dynamics is defined at discrete times from the outset, formulates a discrete variational principle for such dynamics, and then derives a discrete analogue of the Euler-Lagrange or Hamilton's equations from it.

The advantage of this construction is that it naturally gives rise to discrete analogues of the concepts and ideas in continuous time that have the same or similar properties, such as symplectic forms, the Legendre transformation, momentum maps, and Noether's theorem [15]. Whereas the main topic in discrete mechanics is the development of structurepreserving algorithms for Lagrangian and Hamiltonian systems [see, e.g., 15], the theoretical aspects of it are interesting in their own right, and furthermore provide insight into the numerical aspects as well.

Another notable feature of discrete mechanics is that it is a generalization of (nonsingular) discrete optimal control problems. In fact, as stated in Marsden and West [15], discrete mechanics is inspired by discrete formulations of

This work was partially supported by NSF grants DMS-604307, DMS0726263, DMS-0907949, and DMS-1010687.

M. Leok and T. Ohsawa are with Department of Mathematics, University of California, San Diego, 9500 Gilman Drive, La Jolla, California 920930112, mleok@math.ucsd.edu, tohsawa@ucsd.edu

A.M. Bloch is with Department of Mathematics, University of Michigan, 530 Church Street, Ann Arbor, Michigan 48109-1043, ablochaumich. edu optimal control problems (see, e.g., Jordan and Polak [10] and Cadzow [5]).

\section{B. Hamilton-Jacobi Theory}

In classical mechanics [see, e.g., 3; 8; 13; 14], the Hamilton-Jacobi equation is first introduced as a partial differential equation satisfied by the action integral. Specifically, let $Q$ be a configuration space and $T^{*} Q$ be its cotangent bundle, and suppose that $(\hat{q}(s), \hat{p}(s)) \in T^{*} Q$ is a solution of Hamilton's equations

$$
\dot{q}=\frac{\partial H}{\partial p}, \quad \dot{p}=-\frac{\partial H}{\partial q},
$$

where $H: T^{*} Q \rightarrow \mathbb{R}$ is the Hamiltonian of the system. Then calculate the action integral along the solution starting from $s=0$ and ending at $s=t$ with $t>0$ :

$$
S(q, t):=\int_{0}^{t}[\hat{p}(s) \cdot \dot{\hat{q}}(s)-H(\hat{q}(s), \hat{p}(s))] d s,
$$

where $q:=\hat{q}(t)$ and we regard the resulting integral as a function of the endpoint $(q, t) \in Q \times \mathbb{R}_{+}$, where $\mathbb{R}_{+}$is the set of positive real numbers. Then by taking a variation of the endpoint $(q, t)$, one obtains a partial differential equation satisfied by $S(q, t)$ :

$$
\frac{\partial S}{\partial t}+H\left(q, \frac{\partial S}{\partial q}\right)=0
$$

This is the Hamilton-Jacobi $(H-J)$ equation.

Conversely, it is shown that if $S(q, t)$ is a solution of the H-J equation then $S(q, t)$ is a generating function for the family of canonical transformations (or symplectic flow) that describe the dynamics defined by Hamilton's equations. This result is the theoretical basis for the powerful technique of exact integration called separation of variables.

The idea of H-J theory is also useful in optimal control theory [see, e.g., 11]. Namely, the Hamilton-Jacobi equation turns into the Hamilton-Jacobi-Bellman (HJB) equation, which is a partial differential equation satisfied by the optimal cost function. It is also shown that the costate of the optimal solution is related to the solution of the HJB equation.

\section{Discrete Hamilton-Jacobi Theory}

The main objective of this paper is to present a discrete analogue of $\mathrm{H}-\mathrm{J}$ theory within the framework of discrete Hamiltonian mechanics [12].

There are some previous works on discrete-time analogues of the H-J equation, such as Elnatanov and Schiff [6] and Lall and West [12]. Specifically, Elnatanov and Schiff [6] derived an equation for a generating function of 
a coordinate transformation that trivializes the dynamics. This derivation is a discrete analogue of the conventional derivation of the continuous-time $\mathrm{H}-\mathrm{J}$ equation [see, e.g., 13, Chapter VIII]. Lall and West [12] formulated a discrete Lagrangian analogue of the $\mathrm{H}-\mathrm{J}$ equation as a separable optimization problem.

\section{Main Results}

Our work was inspired by the result of Elnatanov and Schiff [6], and further extends the result by developing discrete analogues of results in (continuous-time) H-J theory. Namely, we formulate a discrete analogue of Jacobi's solution, which relates the discrete action sum (see Eq. (3) below) with a solution of the discrete $\mathrm{H}-\mathrm{J}$ equation. Another important result in this paper is a discrete analogue of the H-J theorem, which relates the solution of the discrete H-J equation with the solution of the discrete Hamilton's equations.

We also show that the discrete $\mathrm{H}-\mathrm{J}$ equation is a generalization of the discrete Riccati equation and the Bellman equation (discrete HJB equation). (See Fig. 1.) Specifically, we establish a link with discrete-time optimal control theory, and show that the Bellman equation of dynamic programming follows. This link makes it possible to interpret discrete analogues of Jacobi's solution and the H-J theorem in the optimal control setting. Namely, we show that these results reduce to two well-known results in optimal control theory that relate the Bellman equation with the optimal solution. We also show that the discrete $\mathrm{H}-\mathrm{J}$ equation applied to linear discrete Hamiltonian systems reduces to the discrete Riccati equation. This is again a discrete analogue of the well-known result that the $\mathrm{H}-\mathrm{J}$ equation applied to linear Hamiltonian systems reduces to the Riccati equation [see, e.g., 11, p. 421].
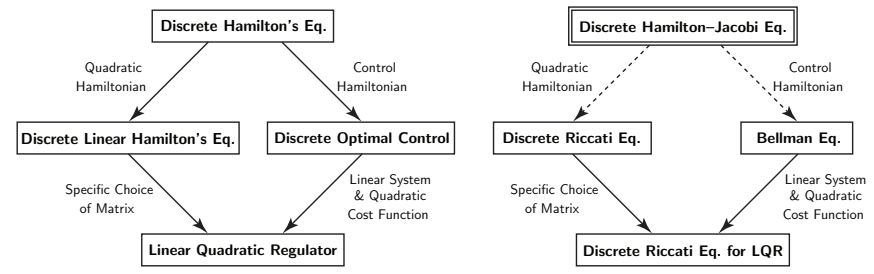

Fig. 1. Discrete evolution equations (left) and corresponding discrete HJ-type equations (right). Dashed lines are the links established in the paper.

\section{E. Outline}

We first present a brief review of discrete Lagrangian and Hamiltonian mechanics in Section II. In Section III we describe a discrete analogue of Jacobi's solution to the discrete $\mathrm{H}-\mathrm{J}$ equation, and also discuss the left and right variants and more explicit forms of the discrete $\mathrm{H}-\mathrm{J}$ equation. In Section IV we prove a discrete version of the $\mathrm{H}-\mathrm{J}$ theorem. Section V establishes the link with discrete-time optimal control and interprets the results of the preceding sections in this setting. In Section VI we apply the theory to linear discrete Hamiltonian systems, and show that the discrete Riccati equation follows from the discrete $\mathrm{H}-\mathrm{J}$ equation.

\section{Discrete MeChANiCS}

This section briefly reviews some key results of discrete mechanics following Marsden and West [15] and Lall and West [12].

\section{A. Discrete Lagrangian Mechanics}

A discrete Lagrangian flow $\left\{q_{k}\right\}$ for $k=0,1, \ldots, N$ on an $n$-dimensional differentiable manifold $Q$ can be described based on the following discrete variational principle. Let $S_{\mathrm{d}}^{N}$ be the following action sum of the discrete Lagrangian $L_{\mathrm{d}}$ : $Q \times Q \rightarrow \mathbb{R}:$

$S_{\mathrm{d}}^{N}\left(\left\{q_{k}\right\}_{k=0}^{N}\right):=\sum_{k=0}^{N-1} L_{\mathrm{d}}\left(q_{k}, q_{k+1}\right) \approx \int_{0}^{t_{N}} L(q(t), \dot{q}(t)) d t$,

where $L: T Q \rightarrow \mathbb{R}$ is the Lagrangian of the corresponding continuous system.

Consider discrete variations $q_{k} \mapsto q_{k}+\epsilon \delta q_{k}$ for $k=$ $0,1, \ldots, N$ with $\delta q_{0}=\delta q_{N}=0$. Then the discrete variational principle $\delta S_{\mathrm{d}}^{N}=0$ gives the discrete Euler-Lagrange equations:

$$
D_{2} L_{\mathrm{d}}\left(q_{k-1}, q_{k}\right)+D_{1} L_{\mathrm{d}}\left(q_{k}, q_{k+1}\right)=0,
$$

where $D_{i}$ stands for the partial derivative with respect to the variable(s) in the $i$-th slot. This determines the discrete flow $F_{L_{\mathrm{d}}}: Q \times Q \rightarrow Q \times Q:$

$$
F_{L_{\mathrm{d}}}:\left(q_{k-1}, q_{k}\right) \mapsto\left(q_{k}, q_{k+1}\right),
$$

\section{B. Discrete Hamiltonian Mechanics}

Lall and West [12] introduced discrete Hamiltonian mechanics in the following way: Introduce the right and left discrete Legendre transforms $\mathbb{F} L_{\mathrm{d}}^{ \pm}: Q \times Q \rightarrow T^{*} Q$ by

$$
\begin{aligned}
& \mathbb{F} L_{\mathrm{d}}^{+}:\left(q_{k}, q_{k+1}\right) \mapsto\left(q_{k+1}, D_{2} L_{\mathrm{d}}\left(q_{k}, q_{k+1}\right)\right), \\
& \mathbb{F} L_{\mathrm{d}}^{-}:\left(q_{k}, q_{k+1}\right) \mapsto\left(q_{k},-D_{1} L_{\mathrm{d}}\left(q_{k}, q_{k+1}\right)\right) .
\end{aligned}
$$

With the discrete Legendre transform

$$
p_{k+1}=\mathbb{F} L_{\mathrm{d}}^{+}\left(q_{k}, q_{k+1}\right)=D_{2} L_{\mathrm{d}}\left(q_{k}, q_{k+1}\right),
$$

we can define the following right discrete Hamiltonian:

$$
H_{\mathrm{d}}^{+}\left(q_{k}, p_{k+1}\right)=p_{k+1} \cdot q_{k+1}-L_{\mathrm{d}}\left(q_{k}, q_{k+1}\right) .
$$

Then the discrete Hamiltonian map $\tilde{F}_{L_{\mathrm{d}}}:\left(q_{k}, p_{k}\right) \mapsto$ $\left(q_{k+1}, p_{k+1}\right)$ is defined implicitly by the right discrete Hamilton's equations

$$
\begin{aligned}
q_{k+1} & =D_{2} H_{\mathrm{d}}^{+}\left(q_{k}, p_{k+1}\right), \\
p_{k} & =D_{1} H_{\mathrm{d}}^{+}\left(q_{k}, p_{k+1}\right) .
\end{aligned}
$$

Similarly, with the discrete Legendre transform

$$
p_{k}=\mathbb{F} L_{\mathrm{d}}^{-}\left(q_{k}, q_{k+1}\right)=-D_{1} L_{\mathrm{d}}\left(q_{k}, q_{k+1}\right),
$$

we can define the following left discrete Hamiltonian:

$$
H_{\mathrm{d}}^{-}\left(p_{k}, q_{k+1}\right)=-p_{k} \cdot q_{k}-L_{\mathrm{d}}\left(q_{k}, q_{k+1}\right) .
$$

Then we have the left discrete Hamilton's equations

$$
\begin{aligned}
q_{k} & =-D_{1} H_{\mathrm{d}}^{-}\left(p_{k}, q_{k+1}\right), \\
p_{k+1} & =-D_{2} H_{\mathrm{d}}^{-}\left(p_{k}, q_{k+1}\right) .
\end{aligned}
$$




\section{DiscRete Hamilton-JACOBI EQUation}

\section{A. Discrete Analogue of Jacobi's Solution}

This section presents a discrete analogue of Jacobi's solution. This also gives an alternative derivation of the discrete $\mathrm{H}-\mathrm{J}$ equation that is much simpler than that of Elnatanov and Schiff [6].

Theorem 1 Consider the action sums Eq. (3) written in terms of the right discrete Hamiltonian, Eq. (8):

$$
S_{\mathrm{d}}^{k}\left(q_{k}\right):=\sum_{l=0}^{k-1}\left[p_{l+1} \cdot q_{l+1}-H_{\mathrm{d}}^{+}\left(q_{l}, p_{l+1}\right)\right]
$$

evaluated along a solution of the right discrete Hamilton's equations (9); each $S_{\mathrm{d}}^{k}\left(q_{k}\right)$ is seen as a function of the end point coordinates $q_{k}$ and the discrete end time $k$. Then these action sums satisfy the right discrete $\mathrm{H}-\mathrm{J}$ equation

$$
\begin{array}{r}
S_{\mathrm{d}}^{k+1}\left(q_{k+1}\right)-S_{\mathrm{d}}^{k}\left(q_{k}\right)-D S_{\mathrm{d}}^{k+1}\left(q_{k+1}\right) \cdot q_{k+1} \\
+H_{\mathrm{d}}^{+}\left(q_{k}, D S_{\mathrm{d}}^{k+1}\left(q_{k+1}\right)\right)=0,
\end{array}
$$

where $D S_{\mathrm{d}}^{k}\left(q_{k}\right)=\left(\partial S_{\mathrm{d}}^{k} / \partial q_{k}^{1}, \ldots, \partial S_{\mathrm{d}}^{k} / \partial q_{k}^{n}\right)$.

Proof: From Eq. (13), we have

$$
S_{\mathrm{d}}^{k+1}\left(q_{k+1}\right)-S_{\mathrm{d}}^{k}\left(q_{k}\right)=p_{k+1} \cdot q_{k+1}-H_{\mathrm{d}}^{+}\left(q_{k}, p_{k+1}\right) \text {, }
$$

where $p_{k+1}$ is considered to be a function of $q_{k}$ and $q_{k+1}$, i.e., $p_{k+1}=p_{k+1}\left(q_{k}, q_{k+1}\right)$. Taking the derivative of both sides with respect to $q_{k+1}$ and using Eq. (12a), we have

$$
p_{k+1}=D S_{\mathrm{d}}^{k+1}\left(q_{k+1}\right) \text {. }
$$

Substituting this into Eq. (15) gives Eq. (14).

Remark 2 Recall that, in the derivation of the continuous $H-J$ equation [see, e.g., 7, Section 23], we consider the variation of the action integral Eq. (1) with respect to the end point $(q, t)$ and find

$$
d S=p d q-H(q, p) d t .
$$

This gives

$$
\frac{\partial S}{\partial t}=-H(q, p), \quad p=\frac{\partial S}{\partial q},
$$

and hence the $H-J$ equation (2). Table I summarizes the correspondence between the ingredients in the continuous and discrete theories (see also Remark 2).

\section{B. The Right and Left Discrete H-J Equations}

We can also write the action sum Eq. (3) in terms of the left discrete Hamiltonian, Eq. (11), as follows:

$$
S_{\mathrm{d}}^{k}\left(q_{k}\right)=\sum_{l=0}^{k-1}\left[-p_{l} \cdot q_{l}-H_{\mathrm{d}}^{-}\left(p_{l}, q_{l+1}\right)\right] .
$$

Then we can proceed as in the proof of Theorem 1 (see Ohsawa et al. [16] for details) to obtain the left discrete $\mathrm{H}-\mathrm{J}$ equation:

$$
\begin{aligned}
S_{\mathrm{d}}^{k+1}\left(q_{k+1}\right)-S_{\mathrm{d}}^{k}\left(q_{k}\right) & +D S_{\mathrm{d}}^{k}\left(q_{k}\right) \cdot q_{k} \\
& +H_{\mathrm{d}}^{-}\left(D S_{\mathrm{d}}^{k}\left(q_{k}\right), q_{k+1}\right)=0 .
\end{aligned}
$$

TABLE I

CORRESPONDENCE BETWEEN INGREDIENTS IN CONTINUOUS AND DISCRETE THEORIES; $\mathbb{R}_{\geq 0}$ IS THE SET OF NON-NEGATIVE REAL NUMBERS AND $\mathbb{N}_{0}$ IS THE SET OF NON-NEGATIVE INTEGERS.

\begin{tabular}{|c|c|}
\hline Continuous & Discrete \\
\hline \hline$(q, t) \in Q \times \mathbb{R}_{\geq 0}$ & $\left(q_{k}, k\right) \in Q \times \mathbb{N}_{0}$ \\
\hline$\dot{q}=\partial H / \partial p$, & $q_{k+1}=D_{2} H_{\mathrm{d}}^{+}\left(q_{k}, p_{k+1}\right)$, \\
$\dot{p}=-\partial H / \partial q$ & $p_{k}=D_{1} H_{\mathrm{d}}^{+}\left(q_{k}, p_{k+1}\right)$ \\
\hline$S(q, t):=\int_{0}^{t} p \dot{q}-H d s$ & $\begin{array}{l}S_{\mathrm{d}}^{k}\left(q_{k}\right):= \\
k-1\end{array}$ \\
$\sum_{l=0}\left[p_{l+1} \cdot q_{l+1}-H_{\mathrm{d}}^{+}\left(q_{l}, p_{l+1}\right)\right]$ \\
\hline$d S=\frac{\partial S}{\partial q} d q+\frac{\partial S}{\partial t} d t$ & $S_{\mathrm{d}}^{k+1}\left(q_{k+1}\right)-S_{\mathrm{d}}^{k}\left(q_{k}\right)$ \\
\hline$p d q-H(q, p) d t$ & $p_{k+1} \cdot q_{k+1}-H_{\mathrm{d}}^{+}\left(q_{k}, p_{k+1}\right)$ \\
\hline$\frac{\partial S}{\partial t}+H\left(q, \frac{\partial S}{\partial q}\right)=0$ & $\begin{array}{c}S_{\mathrm{d}}^{k+1}\left(q_{k+1}\right)-S_{\mathrm{d}}^{k}\left(q_{k}\right) \\
-D S_{\mathrm{d}}^{k+1}\left(q_{k+1}\right) \cdot q_{k+1} \\
+H_{\mathrm{d}}^{+}\left(q_{k}, D S_{\mathrm{d}}^{k+1}\left(q_{k+1}\right)\right)=0\end{array}$ \\
\hline
\end{tabular}

As mentioned above, Eqs. (13) and (19) are the same action sum Eq.(3) expressed in different ways. Therefore we may summarize the above argument as follows:

Proposition 3 The action sums, Eq. (13) or equivalently Eq. (19), satisfy both the right and left discrete $H-J$ equations (14) and (20).

\section{Explicit Forms of the Discrete H-J Equations}

The expressions for the right and left discrete $\mathrm{H}-\mathrm{J}$ equations in Eqs. (14) and (20) are implicit in the sense that they contain two spatial variables $q_{k}$ and $q_{k+1}$. However, Theorem 1 suggests that $q_{k}$ and $q_{k+1}$ may be considered to be related by the dynamics defined by either Eq. (9) or (12). More specifically, we may write $q_{k+1}$ in terms of $q_{k}$. This results in explicit forms of the discrete $\mathrm{H}-\mathrm{J}$ equations, and we shall define the discrete $\mathrm{H}-\mathrm{J}$ equations by the resulting explicit forms.

For the right discrete $\mathrm{H}-\mathrm{J}$ equation (14), we first define the map $f_{k}^{+}: Q \rightarrow Q$ as follows: Replace $p_{k+1}$ in Eq. (9a) by $D S_{\mathrm{d}}^{k+1}\left(q_{k+1}\right)$ as suggested by Eq. (16):

$$
q_{k+1}=D_{2} H_{\mathrm{d}}^{+}\left(q_{k}, D S_{\mathrm{d}}^{k+1}\left(q_{k+1}\right)\right) .
$$

Assuming this equation is solvable for $q_{k+1}$, we define $f_{k}^{+}$: $Q \rightarrow Q$ by the resulting $q_{k+1}$, i.e., $f_{k}^{+}$is implicitly defined by

$$
f_{k}^{+}\left(q_{k}\right)=D_{2} H_{\mathrm{d}}^{+}\left(q_{k}, D S_{\mathrm{d}}^{k+1}\left(f_{k}^{+}\left(q_{k}\right)\right)\right) .
$$

We may now identify $q_{k+1}$ with $f_{k}^{+}\left(q_{k}\right)$ in the implicit form of the right $\mathrm{H}-\mathrm{J}$ equation (14):

$$
\begin{array}{r}
S_{\mathrm{d}}^{k+1}\left(f_{k}^{+}(q)\right)-S_{\mathrm{d}}^{k}(q)-D S_{\mathrm{d}}^{k+1}\left(f_{k}^{+}(q)\right) \cdot f_{k}^{+}(q) \\
+H_{\mathrm{d}}^{+}\left(q, D S_{\mathrm{d}}^{k+1}\left(f_{k}^{+}(q)\right)\right)=0,
\end{array}
$$


where we suppressed the subscript $k$ of $q_{k}$ since it is now clear that $q_{k}$ is an independent variable as opposed to a function of the discrete time $k$. We define Eq. (23) to be the right discrete $H-J$ equation. Notice that these are differentialdifference equations defined on $Q \times \mathbb{N}$, with the spatial variable $q$ and the discrete time $k$.

For the left discrete $\mathrm{H}-\mathrm{J}$ equation (20), we define the map $f_{k}^{-}: Q \rightarrow Q$ as follows:

$$
f_{k}^{-}\left(q_{k}\right):=\pi_{Q} \circ \tilde{F}_{L_{\mathrm{d}}}\left(d S_{\mathrm{d}}^{k}\left(q_{k}\right)\right),
$$

where $\pi_{Q}: T^{*} Q \rightarrow Q$ is the cotangent bundle projection; equivalently, $f_{k}^{-}$is defined so that the diagram below commutes.

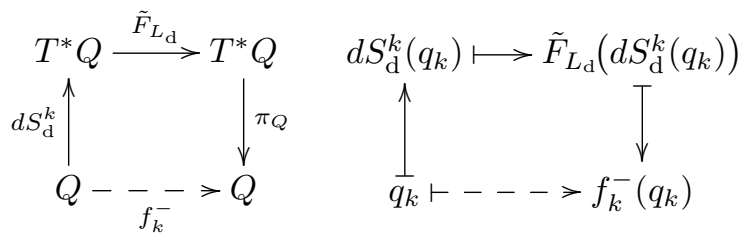

Notice also that, since the map $\tilde{F}_{L_{\mathrm{d}}}:\left(q_{k}, p_{k}\right) \mapsto$ $\left(q_{k+1}, p_{k+1}\right)$ is defined by Eq. (12), $f_{k}^{-}$is defined implicitly by

$$
q_{k}=-D_{1} H_{\mathrm{d}}^{-}\left(D S_{\mathrm{d}}^{k}\left(q_{k}\right), f_{k}^{-}\left(q_{k}\right)\right) .
$$

In other words, replace $p_{k}$ in Eq. (12a) by $D S_{\mathrm{d}}^{k}\left(q_{k}\right)$, and define $f_{k}^{-}\left(q_{k}\right)$ as the $q_{k+1}$ in the resulting equation.

We may now identify $q_{k+1}$ with $f_{k}^{-}\left(q_{k}\right)$ in Eq. (20):

$$
\begin{aligned}
S_{\mathrm{d}}^{k+1}\left(f_{k}^{-}(q)\right)-S_{\mathrm{d}}^{k}(q) & +D S_{\mathrm{d}}^{k}(q) \cdot q \\
& +H_{\mathrm{d}}^{-}\left(D S_{\mathrm{d}}^{k}(q), f_{k}^{-}(q)\right)=0,
\end{aligned}
$$

where we again suppressed the subscript $k$ of $q_{k}$. We define Eqs. (23) and (27) to be the right and left discrete $H-J$ equations, respectively. Notice that these are differentialdifference equations defined on $Q \times \mathbb{N}$, with the spatial variable $q$ and the discrete time $k$.

\section{Discrete H-J Equation and Generating Functions}

Assuming the uniqueness of the solution of the discrete H-J equation, Theorem 1 tells us that, the solution $S_{\mathrm{d}}^{n}(q)$ is the action integral written in terms of the end time and end point, which is the generating function of the the dynamics $\left(q_{0}, p_{0}\right) \mapsto\left(q_{n}, p_{n}\right)$. If we construct $\tilde{F}_{S_{\mathrm{d}}^{n}}$ from $S_{\mathrm{d}}^{n}$ using the correspondence ${ }^{1} L_{\mathrm{d}} \mapsto \tilde{F}_{L_{\mathrm{d}}}$, then $\tilde{F}_{S_{\mathrm{d}}^{n}}$ gives the map $\left(q_{0}, p_{0}\right) \mapsto\left(q_{n}, p_{n}\right)$, i.e., we have

$$
\tilde{F}_{S_{\mathrm{d}}^{n}}=\underbrace{\tilde{F}_{L_{\mathrm{d}}} \circ \cdots \circ \tilde{F}_{L_{\mathrm{d}}}}_{n},
$$

In other words, a solution of the discrete $\mathrm{H}-\mathrm{J}$ equation generates the $n$-step dynamics of the corresponding discrete Lagrangian/Hamiltonian system (see also Fig. 2).

Remark 4 Note that Eq. (28) holds exactly. This property is not guaranteed for those solutions obtained by direct discretizations of the $H-J$ equation.

\footnotetext{
${ }^{1}$ Recall that a discrete Lagrangian is nothing but a generating function. See [15] and [12].
}

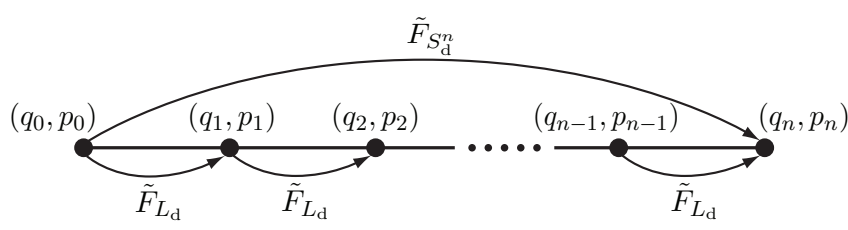

Fig. 2. The generating function $S_{\mathrm{d}}^{n}$ generates the flow defined by the $n$-fold composition $\tilde{F}_{L_{\mathrm{d}}} \circ \cdots \circ \tilde{F}_{L_{\mathrm{d}}}$.

\section{Discrete Hamilton-Jacobi Theorem}

The following gives a discrete analogue of the geometric $\mathrm{H}-\mathrm{J}$ theorem (Theorem 5.2.4) by Abraham and Marsden [1]:

Theorem 5 (Discrete Hamilton-Jacobi) Suppose that $S_{\mathrm{d}}^{k}$ satisfies the right discrete $H-J$ equation (23), and let $\left\{c_{k}\right\}_{k=0}^{N} \subset Q$ be a set of points such that

$$
c_{k+1}=f_{k}^{+}\left(c_{k}\right) \quad \text { for } \quad k=0,1, \ldots, N-1 .
$$

Then the set of points $\left\{\left(c_{k}, p_{k}\right)\right\}_{k=0}^{N} \subset T^{*} Q$ with

$$
p_{k}:=D S_{\mathrm{d}}^{k}\left(c_{k}\right)
$$

is a solution of the right discrete Hamilton's equations (9).

Similarly, suppose that $S_{\mathrm{d}}^{k}$ satisfies the left discrete $H-J$ equation (27), and let $\left\{c_{k}\right\}_{k=0}^{N} \subset Q$ be a set of points that satisfy

$$
c_{k+1}=f_{k}^{-}\left(c_{k}\right) \quad \text { for } \quad k=0,1, \ldots, N-1 .
$$

Furthermore, assume that the Jacobian $D f_{k}^{-}$is invertible at each point $c_{k}$. Then the set of points $\left\{\left(c_{k}, p_{k}\right)\right\}_{k=0}^{N} \subset T^{*} Q$ with

$$
p_{k}:=D S_{\mathrm{d}}^{k}\left(c_{k}\right)
$$

is a solution of the left discrete Hamilton's equations (12).

Proof: See Ohsawa et al. [16].

\section{Relation to the Discrete-Time HJB Equation}

In this section we apply the above results to the optimal control setting. We will show that the (right) discrete $\mathrm{H}-\mathrm{J}$ equation (23) gives the Bellman equation (discrete-time HJB equation) as a special case.

\section{A. Discrete Optimal Control Problem}

Let $\left\{q_{k}\right\}_{k=0}^{N}$ be the state variables in a vector space $V \cong$ $\mathbb{R}^{n}$ with $q_{0}$ and $q_{N}$ fixed and $u_{\mathrm{d}}:=\left\{u_{k}\right\}_{k=0}^{N}$ be controls in the set $U \subset \mathbb{R}^{m}$. With a given function $C_{\mathrm{d}}: V \times U \rightarrow \mathbb{R}$, define the cost functional

$$
J_{\mathrm{d}}:=\sum_{k=0}^{N-1} C_{\mathrm{d}}\left(q_{k}, u_{k}\right) .
$$

Then a typical discrete optimal control problem is formulated as follows [see, e.g., 4; 5; 9; 10]:

Problem 6 Minimize the cost functional, i.e.,

$$
\min _{u_{\mathrm{d}}} J_{\mathrm{d}}=\min _{u_{\mathrm{d}}} \sum_{k=0}^{N-1} C_{\mathrm{d}}\left(q_{k}, u_{k}\right)
$$


subject to the constraint

$$
q_{k+1}=f\left(q_{k}, u_{k}\right) .
$$

B. Necessary Condition for Optimality and the DiscreteTime HJB Equation

We would like to formulate the necessary condition for optimality. First introduce the augmented cost functional:

$$
\begin{aligned}
\hat{S}_{\mathrm{d}}^{k}\left(q_{\mathrm{d}}, p_{\mathrm{d}}, u_{\mathrm{d}}\right) & :=\sum_{l=0}^{k-1}\left\{C_{\mathrm{d}}\left(q_{l}, u_{l}\right)+p_{l+1} \cdot\left[q_{l+1}-f\left(q_{l}, u_{l}\right)\right]\right\} \\
& =\sum_{l=0}^{k-1}\left[p_{l+1} \cdot q_{l+1}-\hat{H}_{\mathrm{d}}^{+}\left(q_{l}, p_{l+1}, u_{l}\right)\right],
\end{aligned}
$$

where we defined the Hamiltonian

$$
\hat{H}_{\mathrm{d}}^{+}\left(q_{l}, p_{l+1}, u_{l}\right):=p_{l+1} \cdot f\left(q_{l}, u_{l}\right)-C_{\mathrm{d}}\left(q_{l}, u_{l}\right)
$$

and the shorthand notation $q_{\mathrm{d}}:=\left\{q_{l}\right\}_{l=0}^{k}, p_{\mathrm{d}}:=\left\{p_{l}\right\}_{l=1}^{k}$, and $u_{\mathrm{d}}:=\left\{u_{l}\right\}_{l=0}^{k-1}$. Then the optimality condition Eq. (34) is restated as $\min _{q_{\mathrm{d}}, p_{\mathrm{d}}, u_{\mathrm{d}}} \hat{J}_{\mathrm{d}}^{k}\left(q_{\mathrm{d}}, p_{\mathrm{d}}, u_{\mathrm{d}}\right)$ or equivalently

$$
\min _{q_{\mathrm{d}}, p_{\mathrm{d}}, u_{\mathrm{d}}} \hat{S}_{\mathrm{d}}^{k}\left(q_{\mathrm{d}}, p_{\mathrm{d}}, u_{\mathrm{d}}\right) \text {. }
$$

In particular, extremality with respect to the control $u_{\mathrm{d}}$ implies

$$
D_{3} \hat{H}_{\mathrm{d}}^{+}\left(q_{l}, p_{l+1}, u_{l}\right)=0, \quad l=0,1, \ldots, k-1 .
$$

Now we assume that $\hat{H}_{\mathrm{d}}^{+}$is sufficiently regular so that the optimal control $u_{\mathrm{d}}^{*}:=\left\{u_{l}^{*}\right\}_{l=0}^{k-1}$ is determined by

$$
D_{3} \hat{H}_{\mathrm{d}}^{+}\left(q_{l}, p_{l+1}, u_{l}^{*}\right)=0, \quad l=0,1, \ldots, k-1 .
$$

Therefore $u_{l}^{*}$ is a function of $q_{l}$ and $p_{l+1}$, i.e., $u_{l}^{*}=$ $u_{l}^{*}\left(q_{l}, p_{l+1}\right)$. Then we can eliminate $u_{\mathrm{d}}$ in the minimization problem Eq. (37):

$$
\min _{q_{\mathrm{d}}, p_{\mathrm{d}}} S_{\mathrm{d}}\left(q_{\mathrm{d}}, p_{\mathrm{d}}\right)=\min _{q_{\mathrm{d}}, p_{\mathrm{d}}} \sum_{l=0}^{k-1}\left[p_{l+1} \cdot q_{l+1}-H_{\mathrm{d}}^{+}\left(q_{l}, p_{l+1}\right)\right],
$$

where we defined $H_{\mathrm{d}}^{+}\left(q_{l}, p_{l+1}\right):=\hat{H}_{\mathrm{d}}^{+}\left(q_{l}, p_{l+1}, u_{l}^{*}\right)$ and $S_{\mathrm{d}}^{k}\left(q_{\mathrm{d}}, p_{\mathrm{d}}\right):=\hat{S}_{\mathrm{d}}^{k}\left(q_{\mathrm{d}}, p_{\mathrm{d}}, u_{\mathrm{d}}^{*}\right)$. So now the problem is reduced to minimizing an action sum that has exactly the same form as the one in Eq. (13) formulated in the framework of discrete Hamiltonian mechanics.

The corresponding right discrete Hamilton's equations are

$$
\begin{aligned}
& q_{k+1}=f\left(q_{k}, u_{k}^{*}\right), \\
& p_{k}=p_{k+1} \cdot D_{1} f\left(q_{k}, u_{k}^{*}\right)-D_{1} C_{\mathrm{d}}\left(q_{k}, u_{k}^{*}\right) .
\end{aligned}
$$

Therefore Eq. (22) gives the implicit definition of $f_{k}^{+}$as follows:

$$
f_{k}^{+}\left(q_{k}\right)=f\left(q_{k}, u_{k}^{*}\left(q_{k}, D S_{\mathrm{d}}^{k+1}\left(f_{k}^{+}\left(q_{k}\right)\right)\right)\right) .
$$

Hence the (right) discrete $\mathrm{H}-\mathrm{J}$ equation (23) applied to this case gives

$$
S_{\mathrm{d}}^{k+1}\left(f\left(q_{k}, u_{k}^{*}\right)\right)-S_{\mathrm{d}}^{k}\left(q_{k}\right)-C_{\mathrm{d}}\left(q_{k}, u_{k}^{*}\right)=0,
$$

or equivalently

$$
\min _{u_{k}}\left[S_{\mathrm{d}}^{k+1}\left(f\left(q_{k}, u_{k}\right)\right)-C_{\mathrm{d}}\left(q_{k}, u_{k}\right)\right]-S_{\mathrm{d}}^{k}\left(q_{k}\right)=0,
$$

which is the Bellman equation [see, e.g., 4].
C. Relation between the Discrete $H-J$ and HJB Equations and its Consequences

Summarizing the observation made above, we have

Proposition 7 The right discrete $H-J$ equation (23) applied to the Hamiltonian formulation of the discrete optimal control problem 6 gives the Bellman equation (44).

Reinterpreting Theorems 1 and 5 in terms of this observation leads to the following well-known facts:

Proposition 8 The optimal cost function satisfies the Bellman equation (44).

Proposition 9 Let $S_{\mathrm{d}}^{k}\left(q_{k}\right)$ be a solution to the Bellman equation (44). Then the costate $p_{k}$ in the discrete maximum principle is given as follows:

$$
p_{k}=D S_{\mathrm{d}}^{k}\left(c_{k}\right),
$$

where $c_{k+1}=f\left(c_{k}, u_{k}^{*}\right)$ with the optimal control $u_{k}^{*}$.

\section{Application To Discrete Linear Hamiltonian SYSTEMS}

A. Discrete Linear Hamiltonian Systems and the Matrix Riccati Equation

\section{Example 10 (Discrete linear Hamiltonian systems)}

Consider a discrete Hamiltonian system on $T^{*} \mathbb{R}^{n} \cong \mathbb{R}^{n} \times \mathbb{R}^{n}$ (the configuration space is $Q=\mathbb{R}^{n}$ ) defined by the quadratic left discrete Hamiltonian

$$
H_{\mathrm{d}}^{-}\left(p_{k}, q_{k+1}\right)=\frac{1}{2} p_{k}^{T} M^{-1} p_{k}+p_{k}^{T} L q_{k+1}+\frac{1}{2} q_{k+1}^{T} K q_{k+1},
$$

where $M, K$, and $L$ are real $n \times n$ matrices; we assume that $M$ and $L$ are invertible and also that $M$ and $K$ are symmetric. The left discrete Hamilton's equations (12) become

$$
\left(\begin{array}{c}
q_{k+1} \\
p_{k+1}
\end{array}\right)=\left(\begin{array}{cc}
-L^{-1} & -L^{-1} M^{-1} \\
K L^{-1} & K L^{-1} M^{-1}-L^{T}
\end{array}\right)\left(\begin{array}{c}
q_{k} \\
p_{k}
\end{array}\right) .
$$

Now let us solve the left discrete H-J equation (27) for this system. It is possible to show (see Ohsawa et al. [16]) that, for this particular case, the solution $S_{\mathrm{d}}^{k}$ takes the form

$$
S_{\mathrm{d}}^{k}(q)=\frac{1}{2} q^{T} A_{k} q+b_{k}^{T} q+c_{k}
$$

where $A_{k}$ are symmetric $n \times n$ matrices, $b_{k}$ are elements in $\mathbb{R}^{n}$, and $c_{k}$ are in $\mathbb{R}$. We substitute the above expression into the discrete $H-J$ equation to find the equations for $A_{k}, b_{k}$, and $c_{k}$. Notice first that the map $f_{k}^{-}$is given by the first half of Eq. (47) with $p_{k}$ replaced by $D S_{\mathrm{d}}^{k}(q)$ :

$$
f_{k}^{-}(q)=-L^{-1}\left(I+M^{-1} A_{k}\right) q-L^{-1} M^{-1} b_{k} .
$$

Then substituting Eq. (48) into the left-hand side of the left discrete H-J equation (27) yields the following recurrence 
relations for $A_{k}, b_{k}$, and $c_{k}$ :

$$
\begin{aligned}
A_{k+1} & =L^{T}\left(I+A_{k} M^{-1}\right)^{-1} A_{k} L-K, \\
b_{k+1} & =-L^{T}\left(I+A_{k} M^{-1}\right)^{-1} b_{k}, \\
c_{k+1} & =c_{k}-\frac{1}{2} b_{k}^{T}\left(M+A_{k}\right)^{-1} b_{k},
\end{aligned}
$$

where we assumed that $I+A_{k} M^{-1}$ is invertible.

Remark 11 For the $A_{k+1}$ defined by Eq. (50a) to be symmetric, it is sufficient that $A_{k}$ is invertible; for if it is, then Eq. (50a) becomes

$$
A_{k+1}=L^{T}\left(A_{k}^{-1}+M^{-1}\right)^{-1} L-K,
$$

where $A_{k}, M$, and $K$ are symmetric.

Remark 12 We can rewrite Eq. (50a) as follows:

$$
\begin{aligned}
A_{k+1}=\left[K L^{-1}+\right. & \left.\left(K L^{-1} M^{-1}-L^{T}\right) A_{k}\right] \\
& \times\left(-L^{-1}-L^{-1} M^{-1} A_{k}\right)^{-1} .
\end{aligned}
$$

Notice the exact correspondence between the coefficients in the above equation and the matrix entries in the discrete linear Hamiltonian equations (47). In fact, this is the discrete Riccati equation that corresponds to the iteration defined by Eq. (47). See Ammar and Martin [2] for details on this correspondence.

To summarize the above observation, we have

Proposition 13 The discrete $H_{-} J$ equation (27) applied to the discrete linear Hamiltonian system (47) yields the discrete Riccati equation (51).

In other words, the discrete $\mathrm{H}-\mathrm{J}$ equation is a nonlinear generalization of the discrete Riccati equation.

\section{CONCLUSION AND FUtURE WORK}

We developed a discrete-time analogue of the H-J theory starting from the discrete variational Hamilton equations formulated by Lall and West [12]. We showed that it possesses theoretical significance in discrete mechanics that is equivalent to that of the (continuous-time) $\mathrm{H}-\mathrm{J}$ equation in Hamiltonian mechanics. Furthermore, we showed that the discrete $\mathrm{H}-\mathrm{J}$ equation specializes to the Bellman equation if applied to discrete optimal control problems, and also that it reduces to the discrete Riccati equation with a quadratic Hamiltonian. This again gives discrete analogues of the corresponding known results in the continuous-time theory. Application to discrete optimal control also revealed that Theorems 1 and 5 specialize to two well-known results in discrete optimal control theory.

We are interested in the following topics for future work: (i) Application to integrable discrete systems; (ii) Development of numerical methods based on the discrete $\mathrm{H}-\mathbf{J}$ equation; (iii) Extension to discrete nonholonomic and Dirac mechanics; (iv) Relation to the power method and iterations on the Grassmannian manifold.

\section{ACKNOWLEDGMENTS}

We would like to thank Jerrold Marsden, Harris McClamroch, Matthew West, Dmitry Zenkov, and Jingjing Zhang for helpful discussions and comments.

\section{REFERENCES}

[1] R. Abraham and J. E. Marsden. Foundations of Mechanics. Addison-Wesley, 2nd edition, 1978.

[2] G. Ammar and C. Martin. The geometry of matrix eigenvalue methods. Acta Applicandae Mathematicae, 5(3):239-278, 1986.

[3] V. I. Arnold. Mathematical Methods of Classical Mechanics. Springer, 1989.

[4] R. Bellman. Introduction to the Mathematical Theory of Control Processes, volume 2. Academic Press, 1971.

[5] J. A. Cadzow. Discrete calculus of variations. International Journal of Control, 11(3):393-407, 1970.

[6] N. A. Elnatanov and J. Schiff. The Hamilton-Jacobi difference equation. Functional Differential Equations, 3(279-286), 1996.

[7] I. M. Gelfand and S. V. Fomin. Calculus of Variations. Dover, 2000.

[8] H. Goldstein, C. P. Poole, and J. L. Safko. Classical Mechanics. Addison Wesley, 3rd edition, 2001.

[9] V. Guibout and A. M. Bloch. A discrete maximum principle for solving optimal control problems. In $43 \mathrm{rd}$ IEEE Conference on Decision and Control, volume 2, pages 1806-1811 Vol.2, 2004.

[10] B. W. Jordan and E. Polak. Theory of a class of discrete optimal control systems. Journal of Electronics and Control, 17:694-711, 1964.

[11] V. Jurdjevic. Geometric control theory. Cambridge University Press, Cambridge, 1997.

[12] S. Lall and M. West. Discrete variational Hamiltonian mechanics. Journal of Physics A: Mathematical and General, 39(19):5509-5519, 2006.

[13] C. Lanczos. The Variational Principles of Mechanics. Dover, 4th edition, 1986.

[14] J. E. Marsden and T. S. Ratiu. Introduction to Mechanics and Symmetry. Springer, 1999.

[15] J. E. Marsden and M. West. Discrete mechanics and variational integrators. Acta Numerica, pages 357-514, 2001.

[16] T. Ohsawa, A. M. Bloch, and M. Leok. Discrete Hamilton-Jacobi theory. Preprint (arXiv:0911.2258).

[17] Y. B. Suris. The problem of integrable discretization: Hamiltonian approach. Birkhäuser, Basel, 2003.

[18] Y. B. Suris. Discrete Lagrangian models. In Discrete Integrable Systems, volume 644 of Lecture Notes in Physics, pages 111-184. Springer, 2004. 